\title{
Numerical simulation of a heat generation in a layered material during ultrasonic wave propagation
}

\author{
Anastasiia Kostina \\ Research and Education Center "Materials", Don State Technical University, Rostov-on-Don, Russia \\ kostina@icmm.ru, http:/ /orcid.org/0000-0002-5721-3301 \\ Oleg Plekhov \\ Institute of Continuous Media Mechanics of the Ural Branch of Russian Academy of Sciences, Perm, Russia \\ poa@icmm.ru, bttp:/ orcid.org/ 0000-0002-0378-8249
}

Sergey Aizikovich

Research and Education Center "Materials", Don State Technical University, Rostov-on-Don, Russia

saizikovich@gmail.com, bttp:/ / orcid.org/0000-0002-2756-5752

\begin{abstract}
Ultrasonic vibrothermography is an effective and non-destructive method, which can be used for a quick detection of coating defects on large surfaces. Mechanical excitation of a layered structure induces inelastic deformation and, as a consequence, energy dissipation in the defective area and local heating. In this work, a three-dimensional numerical simulation of this process is applied to the bi-metallic layered structure with an edge crack. Two models of energy dissipation are considered (hysteretic damping model and visco-elastic Maxwell's model) for simulation of energy dissipation in a crack tip area under ultrasonic loading. The models allow us to study the effects of the loading frequency, loading direction and location of the coating defects on the heat dissipation and propose optimal regimes for the ultrasonic vibrothermography of bi-metallic layered structures.
\end{abstract}

KEYwORDS. Ultrasonic thermography; Non-destructive technique; Energy dissipation; Finite-element analysis.

\section{OPEN ACCESS}

Citation: Kostina, A., Plekhov, O., Aizikovich, S., Numerical simulation of a heat generation in a layered material during ultrasonic wave propagation, Frattura ed Integrità Strutturale, 46 (2018) 332-342.

Received: 08.08 .2018

Accepted: 10.09 .2018

Published: 01.10 .2018

Copyright: (C) 2018 This is an open access article under the terms of the CC-BY 4.0, which permits unrestricted use, distribution, and reproduction in any medium, provided the original author and source are credited.

\section{INTRODUCTION}

$\mathrm{U}$ ltrasonic vibrothermography is a safe, non-destructive technique, which can be applied to the detection of defects in metals and composite materials [1-3]. This method is an effective way to study characteristics of the coating and monitor hidden defects. The loading frequency, loading direction and location of the coating defects 
play a key role in the energy dissipation under ultrasonic loading. To increase the efficiency of application of ultrasonic vibrothermography method for layered structures it is necessary to develop an adequate mathematical model, which can predict the value of energy dissipation and give one an opportunity to optimize the monitoring procedure without expensive experimental program.

If solids are perfectly elastic then there is no heat dissipation. Such solids are characterized by linear relation between stress and strain values during the whole process of excitation. Heat dissipation in elastic wave take place if stress and strain are not related by a proportional dependence during the period of vibration. In this case, the hysteresis effect and attenuation of the vibrational amplitude are observed.

In viscoelastic media there are several sources of damping. The main of them are dissipation due to the nonlinear relation between stresses and strains, frictional rubbing of contact surfaces and thermoelastic damping [4]. The nature of thermoelastic damping is related to the fact that when the solid is subjected to the loading, the arising strain leads to the temperature increase. If the strain is homogeneous along the specimen, the change in the temperature value is distributed uniformly. Inhomogeneous strain distribution (for example, in the presence of the defect) leads to the local temperature gradients and irreversible processes due to heat conductivity. Therefore, the heat generation occurs in the specimen containing defects. In solids, thermoelastic effect is small compared to the other (inelastic) mechanisms of damping [4]. and it is neglected in this work. Heat generation in cracks can be explained by frictional heating arising from rubbing contact surfaces. Therefore, the crack can be considered as a local heat source.

In recent years, much works have been done to simulate dissipation during the ultrasonic wave propagation. Rizi et al. [5] carried out numerical simulation of crack heating during ultrasound wave propagation. They have shown that crack length has a negligible effect on a hysteresis heating. Amount of heat dissipated by friction depends on the size of the crack, gap between crack surface, sliding velocity and contact pressure. In [6] thermal response of the crack is evaluated by numerical scheme based on the solution to the contact problem. The main feature of the proposed approach is simulation of the rough crack surfaces allowing the description of a non-uniformly distributed heating. S.R. Hiremath et al. [7] evaluated temperature rise and velocity in linear elastic plate with a crack. It has been shown that presence of the crack induces deviation of the wave velocity and temperature from their values obtained for the case of an ideal plate without crack. Analytic relation describing temperature rise in a flat-bottom hole during the ultrasonic excitation was obtained in [8]. The proposed solution was based on the concept of a local defect resonance. Calculations and experimental results confirm that the use of this concept strongly intensifies local vibrations and enhances efficiency of vibrothermography.

An overview of the existing works shows that defect detection by thermosonic technique can be successfully modelled by a finite-element method. However, most of the considered works are devoted to the application of vibrothermography method to the crack location in a one-layered material. In this work, simulation of the thermosonic method is carried out for a two-layered material (steel specimen with a viscoelastic thin damaged coating). The effect of the loading frequency on the heat dissipation value as well as loading direction and location of the coating defects are investigated in the framework of this work. We have considered an ultrasonic excitation of a bi-metallic sample which include elastic body (steel) coated by viscoelastic media (copper). The steel is considered as a linear elastic solid while an inelastic thin layer with geometric shortcoming models the coating. A coupled thermo-mechanical model is used for the calculation of the heat dissipation. Internal losses in the considered system "elastic solid-viscoelastic coating" are induced by the dissipation of a mechanical energy. In this work, two models of energy dissipation are formulated (hysteretic damping model and visco-elastic Maxwell's model). Results obtained by the model is compared to results reported in [5]. The obtained results can be used for an optimal choice of the ultrasonic vibrothermography parameters and substitution of expensive experimental procedures.

\section{MATERIAL \& METHOD}

he main idea of the thermosonic technique is to use high-frequency vibrations for local excitation of the defects. Coating defects are passive in the sense that they have a uniform temperature equal to the ambient temperature and require additional mechanical or thermal excitation in order to obtain a useful temperature signal. Therefore, the specimen with the damaged coating is subjected to the ultrasonic vibration with the loading frequency which usually lies in the range from 10000 to $60000 \mathrm{~Hz}$. Cracks or other structural defects induce local heating of the specimen which is identified by the infrared thermography systems. However, the applicability of this technique to the real structures requires additional investigations. The numerical simulation is a powerful tool which can be used for an optimal choice of such technological parameters as loading frequency or loading direction for the structures with the specific geometry. 
In this work, a finite-element simulation of the thermosonic method has been carried out to determine the local heating of the bi-metallic specimen (steel sample coated by a thin layer of copper) with an edge crack. The sample is considered as a linear elastic solid while the coating is modelled as a visco-elastic media. Thermophysical and mechanical parameters of the materials are presented in Tabs. 1 and 2.

\begin{tabular}{cccc}
\hline Material & $\begin{array}{c}\text { Heat capacity, } \\
\mathrm{J} /(\mathrm{kg} * \mathrm{~K})\end{array}$ & $\begin{array}{c}\text { Thermal } \\
\text { conductivity, } \\
\mathrm{W} /(\mathrm{m} * \mathrm{~K})\end{array}$ & Density, $\mathrm{kg} / \mathrm{m} 3$ \\
Copper & 385 & 401 & 8900 \\
Steel & 502 & 45.4 & 7870 \\
\hline
\end{tabular}

Table 1: Thermophysical properties of the specimen and coating.

\begin{tabular}{ccccccc}
\hline Material & $\begin{array}{c}\text { Young's } \\
\text { modulus, Pa }\end{array}$ & Poisson's ratio & $\begin{array}{c}\text { Thermal } \\
\text { expansion } \\
\text { coefficient, } \\
1 / \mathrm{K}\end{array}$ & $\begin{array}{c}\text { Shear } \\
\text { modulus, Pa }\end{array}$ & $\begin{array}{c}\text { Isotropic loss } \\
\text { factor }\end{array}$ & $\begin{array}{c}\text { Relaxation } \\
\text { time, s }\end{array}$ \\
Copper & $10^{11}$ & 0.35 & $16.7 * 10^{-6}$ & $3.7 * 10^{10}$ & $10^{-4}$ & $5.5^{*} 10^{-10}$ \\
Steel & $2.13^{*} 10^{11}$ & 0.28 & $11.9 * 10^{-6}$ & - & - & - \\
\hline
\end{tabular}

Table 2: Mechanical properties of the specimen and coating.

\section{THEORY}

$\mathrm{P}$

ropagation of elastic waves in an isotropic media is described by the differential equation of motion, which has the following form:

$$
\rho \frac{\partial^{2} \mathbf{u}}{\partial t^{2}}=\nabla \cdot \sigma+\mathbf{f}
$$

where $\rho$ is the density, $\mathbf{u}$ is the displacement vector, $t$ is the time, $\sigma$ is the Cauchy's stress tensor, $\mathbf{f}$ is the volumetric force. Differential Eqn. (1) can be transformed into the algebraic in the frequency domain with the use of Fourier transform of time derivative [9]. According to the differentiation theorem, Fourier transform of the second time derivative can be expressed as

$$
F\left[\frac{d^{2}}{d t^{2}} \mathbf{u}(t)\right]=-\omega^{2} F[\mathbf{u}(t)]
$$

where $F[f(t)]=\int_{-\infty}^{+\infty} f(t) \mathrm{e}^{-i \omega t} d t$ is Fourier transform of a function $f(t), \omega$ is the angular frequency, $i$ is the imaginary unit. Application of Fourier transformation together with the differentiation theorem to (1) give the representation of (1) in the form:

$$
-\rho \omega^{2} \mathbf{u}=\nabla \cdot \sigma+\mathbf{F} e^{i \varphi}
$$

where $\varphi$ is the phase.

For an elastic solid the relation between stress and strain tensor components can be expressed in the form of the Hook's law:

$$
\sigma=\mathbf{C}: \varepsilon^{d l}
$$


where $\mathbf{C}$ is the fourth-order stiffness tensor, $\boldsymbol{\varepsilon}^{e l}$ is the elastic strain tensor. In case of the isotropic material, the stiffness tensor has two components:

$$
\mathbf{C}=\mathbf{C}(E, v)
$$

where $E$ is the Young's modulus, $v$ is the Poisson's ratio.

Volumetric strain due to a thermal expansion is calculated as:

$$
\boldsymbol{\varepsilon}^{T}=\alpha\left(T-T_{0}\right) \mathbf{E}
$$

where $\alpha$ is linear thermal expansion coefficient, $T$ is the temperature, $T_{0}$ is the initial temperature value.

In case of the small strains, full strain tensor $\varepsilon=\varepsilon^{e}+\varepsilon^{T}$ is related to the displacement vector $\mathbf{u}$ by the geometric equation:

$$
\varepsilon=\frac{1}{2}\left(\nabla \mathbf{u}+\nabla \mathbf{u}^{T}\right)
$$

A perfect elastic body doesn't have heat losses. This solid can be described by a linear relation (4). However, a real process of distribution of high-frequency elastic waves in solids is always accompanied by the energy dissipation related to the irreversible processes of internal friction (viscosity), thermal conductivity and diffusion.

Intrinsic friction in the material is connected to the dissipative processes, which take place during the mechanical vibration of the system. Diversity of dissipative processes in the material has led to the large number of models taking into account energy dissipation during the dynamic loading. These models can be conditionally divided into two classes. The first class includes non-linear models describing hysteretic damping during the cyclic loading. A cyclic vibrational loading leads to the lag of the strain with respect to the stress (stress and strain are not in phase) and the attenuation of the elastic wave takes place. The second class includes models describing viscoelastic behavior of the material during the deformation process. In this work, both classes of the models are considered.

A visco-elastic behavior of the coating is described by the Maxwell's model. In the simplest one-dimensional case, this model can be represented as the sequential connection of an elastic spring and a viscous damper. The form of this constitutive equation in the frequency domain according to [10] is given below.

In the frequency domain, the following representations of the strain and stress tensors are valid:

$$
\begin{aligned}
& \sigma_{d}^{\prime}=\operatorname{real}\left(\overline{\boldsymbol{\sigma}}_{d} e^{i \omega t}\right) \\
& {\boldsymbol{\varepsilon}_{d}^{\prime}}_{d}=\operatorname{real}\left(\overline{\boldsymbol{\varepsilon}}_{d} e^{i \omega t}\right)
\end{aligned}
$$

where $\bar{\sigma}_{d}=\sigma_{d} e^{i \varphi}$ and $\bar{\varepsilon}_{d}=\varepsilon_{d} e^{i \varphi}, \sigma_{d}$ is the deviatoric stress tensor, $\varepsilon_{d}$ is the deviatoric strain tensor.

Then, the Hook's law for the deviatoric stress tensor can be written as

$$
\boldsymbol{\sigma}_{d}^{\prime}=2\left(G^{\prime}+i G^{\prime \prime}\right) \boldsymbol{\varepsilon}_{d}^{e /}
$$

where $G^{\prime}=G_{v} \frac{\left(\omega \tau_{v}\right)^{2}}{1+\left(\omega \tau_{v}\right)^{2}}$ is the shear storage modulus, $G^{\prime \prime}=G_{v} \frac{\omega \tau_{v}}{1+\left(\omega \tau_{v}\right)^{2}}$ is the shear loss modulus, $\tau_{v}$ is the relaxation time, $G_{v}$ is the shear modulus.

Energy dissipation averaged over the time period $2 \pi / \omega$ is expressed as

$$
Q_{v}=\omega G^{\prime \prime} \bar{\varepsilon}_{d}: \operatorname{conj}\left(\bar{\varepsilon}_{d}\right)
$$

where $\operatorname{conj}(\cdot)$ - complex conjugate values.

Material (hysteretic) damping can be described by an isotropic loss factor $\eta$ which is added to the elastic stiffness matrix: 


$$
\mathbf{C}^{\prime}=(1+i \eta) \mathbf{C}
$$

In this case, inelastic work of viscous forces averaged over the time $2 \pi / \omega$ can be calculated as

$$
Q_{b}=\frac{1}{2} \omega \eta \operatorname{real}(\varepsilon: \operatorname{conj}(\mathbf{C}: \varepsilon))
$$

Therefore, energy dissipation in this work is described by two mechanisms: intrinsic damping in the main material (hysteretic damping) $Q_{b}$ (13) and viscous mechanical dissipation $Q_{v}(11)$.

Temperature distribution in the specimen is calculated according to the heat transfer equation with heat sources induced by material damping or viscous behavior of the material:

$$
\rho C_{p} \frac{\partial T}{\partial t}+\nabla \cdot(-k \nabla T)=\mathcal{Q}
$$

where $T$ is the temperature, $C_{p}$ is the heat capacity under the constant pressure, $k$ is the thermal conductivity, $Q$ is the heat source.

Eqns. (3)-(7), (10)-(11), (14) were used for the simulation of a temperature rise induced by the viscous behavior of the coating while the Eqns. (3)-(7), (12)-(14) were applied to the simulation of the material damping. Boundary conditions are specified individually for each considered case and given in the section below.

\section{RESULTS OF NUMERICAL SIMULATION \& DISCUSSION}

\section{Model verification}

he data reported in [5] were used in order to verify the correctness of the presented model. For this purpose, a finite-element simulation of the heat generation in a damaged aluminum plate was performed. The plate has dimensions of 150x150x3 mm and contains an edge crack with a length of $20 \mathrm{~mm}$. The mechanical and thermophysical properties of the material have been taken from [5]. It is assumed that the load is applied on a circular surface with a radius of $5 \mathrm{~mm}$ located at the center of the plate. The loading direction is perpendicular to the plate, has a frequency of $20000 \mathrm{~Hz}$ and the amplitude of $0.05 \mathrm{~mm}$. The duration of the loading is 1 second. A crack tip is modelled as an area with an initial strain localization due to the presence of the large number of microdefects. The initial strain amplitude at the crack tip is equal to 0.165 , the isotropic loss factor is set to $0.45 \cdot 10^{-6}$. The COMSOL Multiphysics ${ }^{\circledR}$ software is used to perform the modeling.

To calibrate the size of the mesh, the convergence study has been performed. The considered area was discretized by a number of tetrahedral finite elements having various sizes. A more refined mesh at the crack tip was used. The maximum element size was varied from $45 \mathrm{~mm}$ to $2 \mathrm{~mm}$. A temperature increase at the crack tip has been obtained as a result of each simulation and the relative error has been calculated. The convergence plot is presented in fig. 1 (a). It can be concluded that the element size should be smaller than $5 \mathrm{~mm}$ because in this case the relative error does not exceed $15 \%$. A finiteelement mesh with the maximum element size of $2 \mathrm{~mm}$ has been chosen to carry out numerical simulation.

Fig. 1 (b) shows a comparison of the obtained result to the data presented in [5]. The obtained results show that the model can describe adequately the temperature rise at the crack tip.

\section{Numerical simulation of a heat generation in a layered material with coating defects}

In this section an ultrasonic vibration of a rectangular steel bar (the sizes of the bar are 200x10x3 mm) with a copper coating of a $50 \mu \mathrm{m}$ thickness is considered. The coating of the specimen has an edge crack with a length of $3 \mathrm{~mm}$ and a depth of $50 \mu \mathrm{m}$, which is simulated as an acute-angled notch. Schematic representation of the specimen is presented in fig. 2. The considered area was discretized by a number of tetrahedral finite elements. The convergence analysis has shown that the length of the element should not exceed $5 \mathrm{~mm}$. Therefore, a finite-element mesh with a maximum element length of $1.8 \mathrm{~mm}$ and a minimal size of $0.408 \mathrm{~mm}$ was used (fig. 3). This fine resolution provides an accurate simulation of a heat localization at the crack tip. A total number of the finite elements was approximately 250000 . 
The sample is considered as a perfectly elastic isotropic solid which has no energy dissipation. The coating is considered as a visco-elastic material subjected to the Maxwell's model or as isotropic elastic solid with a material damping describing by an isotropic loss factor. The crack tip is simulated as an area with an initial strain localization and, as a consequence, with a large number of microdefects.

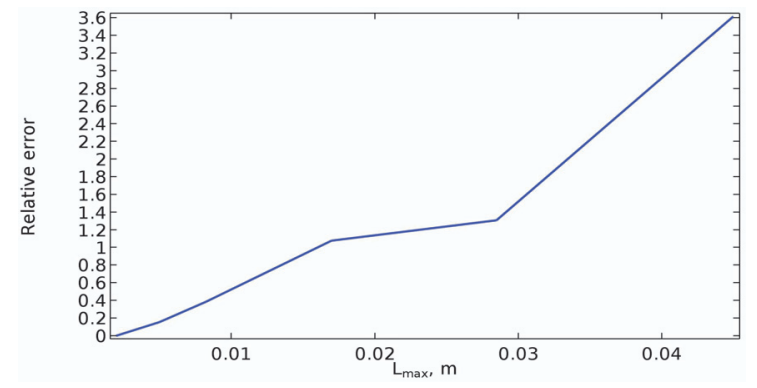

(a)

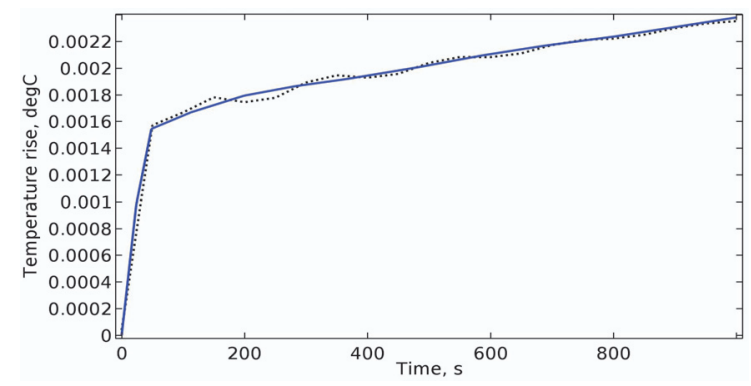

(b)

Figure 1: (a) A relative error versus maximum size of the element. (b) Temperature rise at edge crack tip (solid line - results presented in [5], dashed line - results of the developed model).

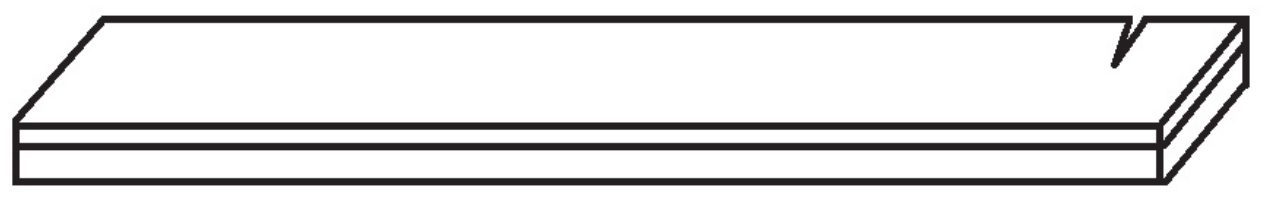

Figure 2: Schematic representation of the two-layered specimen.

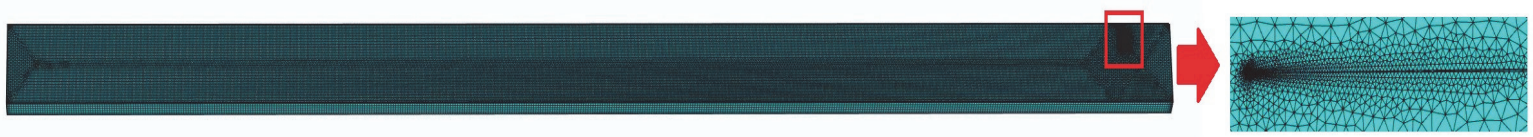

Figure 3: Finite-element mesh of the two-layered specimen.

It is supposed that the specimen is laid on some surface during the loading process. Therefore, fixed constrained boundary conditions were set to the lower boundary. The initial temperature of the sample is assumed to be $293.15 \mathrm{~K}$. Convective heat flux with a heat transfer coefficient equal to $6 \mathrm{~W} /\left(\mathrm{m}^{2 *} \mathrm{~K}\right)$ was applied to all boundaries of the specimen except for the lower. The lower boundary is assumed to be thermally insulated.

Fig. 4 presents results of continuous ultrasonic excitation of the sample by a point load applied to the side of the sample opposite to a crack location obtained by two models: material damping and visco-elastic Maxwell's model. The loading direction was perpendicular to the crack plane, the applied loading frequency was $20000 \mathrm{~Hz}$, the duration of the loading was 1 second. It can be concluded that the crack location can be sharply visible in both models. The main difference is that damping model also made visible the point of load application.

Fig. 5 shows evolution of the temperature rise during the loading process. The maximum value of the temperature is observed at the crack tip. On the initial stage of the excitation, the rate of the temperature rise is quite large. At subsequent stages of excitations, it is observed a decrease in the temperature rise rate induced by the attenuation of the wave. These results are confirmed by Fig. 6 (a). It can be seen that there is only a slight increase in the temperature rise value in the end of the excitation process at the crack tip. Moreover, fig. 6 (a) shows that the applied load and the used values of material parameters give the same temperature rise at the crack tip. This result can be interpreted as the equivalence of the damping model and visco-elastic model under the considered conditions. Numerical simulation has shown also that the choice of material parameters for both models affect the sensitivity of the obtained results to the applied load. A decrease in the isotropic loss factor value and relaxation time value by an order of magnitude gives a sensitivity of the temperature rise by the loading magnitude. Fig. 6 (b) presents results of ultrasonic excitation of the end face of the specimen by various surface loads. The graphs show that the sensitivity of the damping model is slightly larger than the Maxwell's model. However, values of the isotropic loss factor and relaxation time given in the Tab., 2 are insensitive to the applied load. 


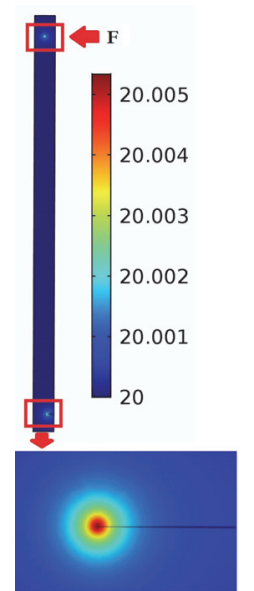

(a)

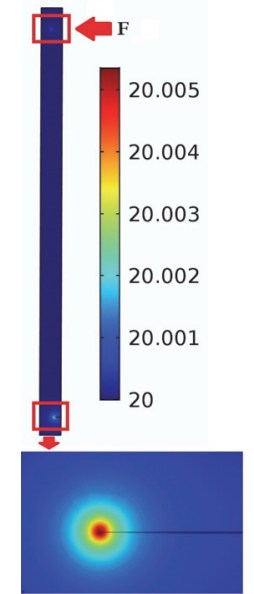

(b)

Figure 4: Temperature distribution $\left(\mathrm{C}^{\circ}\right)$ in the specimen after ultrasonic excitation after 1 second of loading obtained by two models: (a) material damping, (b) visco-elastic Maxwell's model.

Temperature rise, deg C; time $=0.3 \mathrm{~s}$

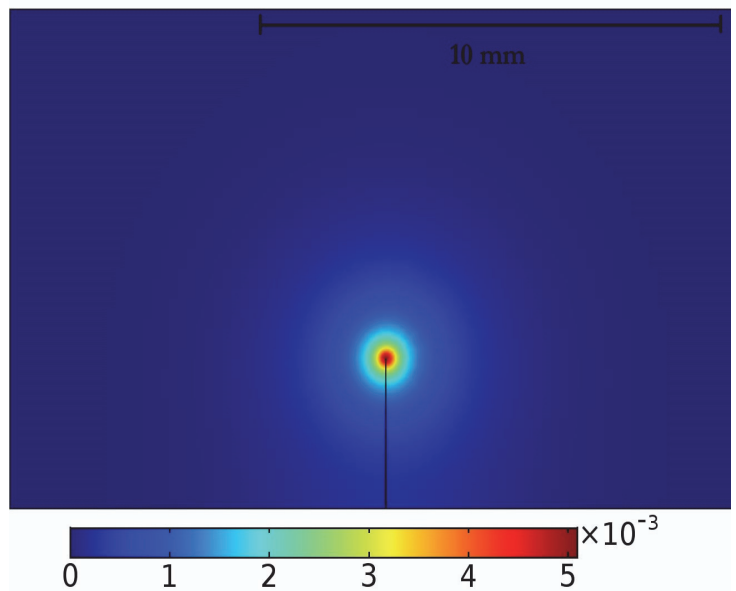

(a)

Temperature rise, $\operatorname{deg} \mathrm{C}$; time $=0.7 \mathrm{~s}$

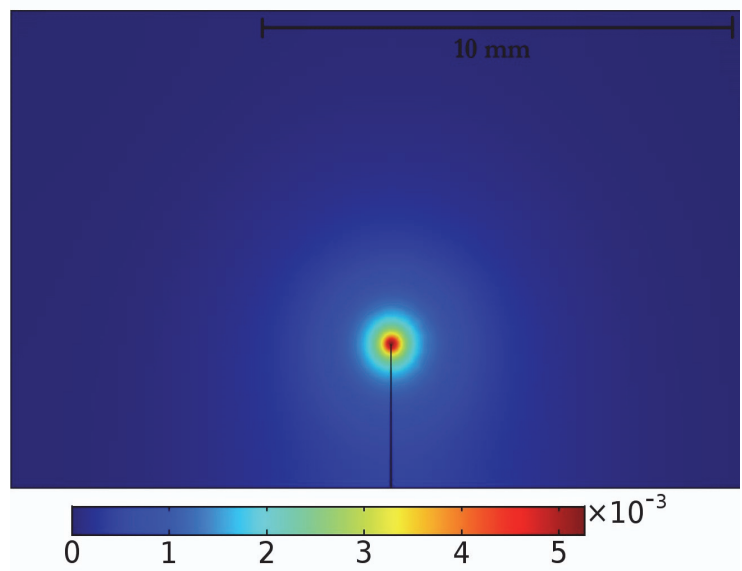

(c)
Temperature rise, $\operatorname{deg} C$; time $=0.1 \mathrm{~s}$
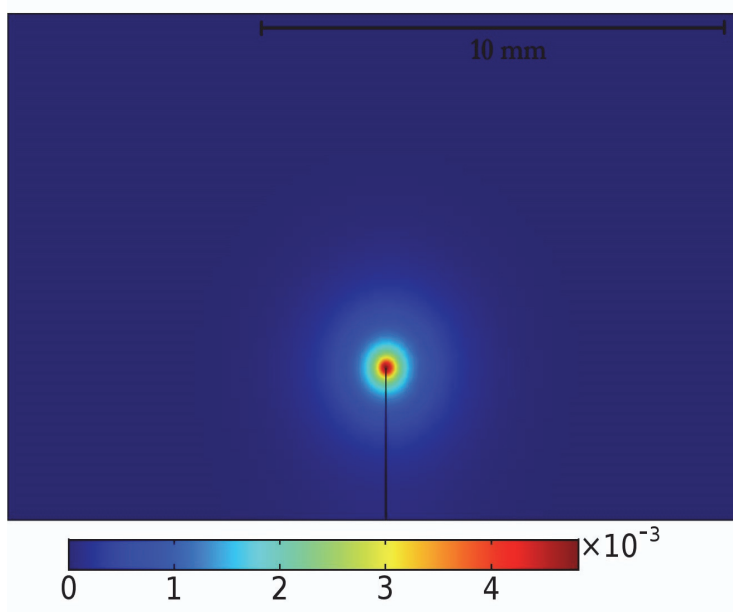

(b)

Temperature rise, deg C; time $=1 \mathrm{~s}$

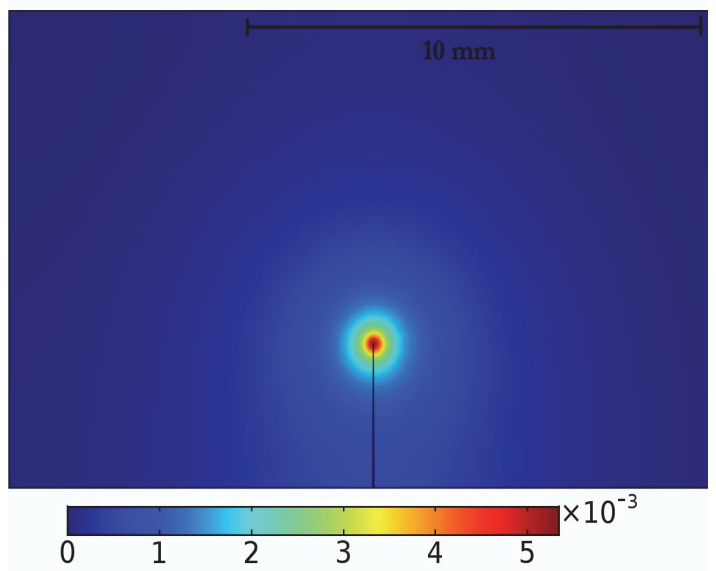

(d)

Figure 5: Temperature rise near the crack tip in different moments of loading time obtained under frequency equal to $20000 \mathrm{~Hz}$ : (a) $\mathrm{t}=0.1$ second, (b) $\mathrm{t}=0.3$ second, (c) $\mathrm{t}=0.7$ second, (d) $\mathrm{t}=1$ second. 


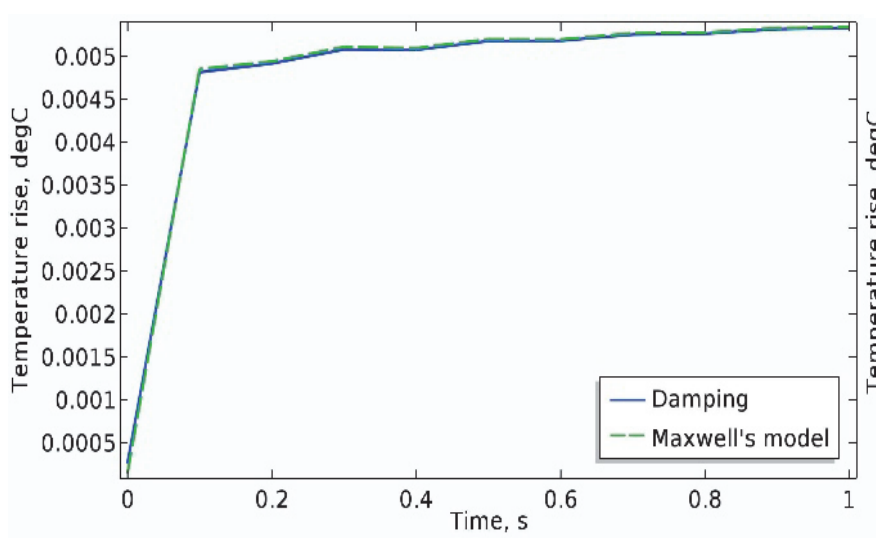

(a)

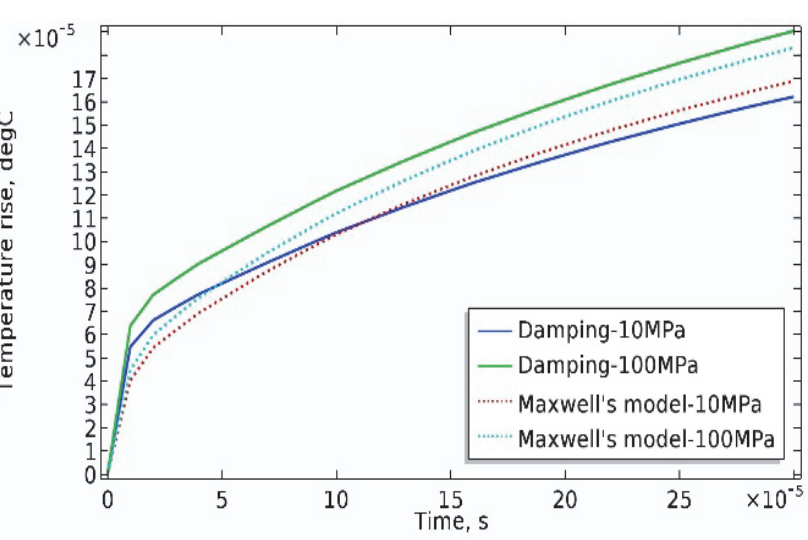

(b)

Figure 6: Temperature rise at the crack obtained by isotropic damping model and Maxwell's model: (a) $\eta=10^{-4}, \tau_{v}=5.5^{*} 10^{-10} \mathrm{~s}(\mathrm{solid}$ line - isotropic damping model, dashed line - visco-elastic Maxwell's model); (b) $\eta=10^{-5}, \tau_{v}=5.5^{*} 10^{-11} \mathrm{~s}$ (solid green line - damping model, the magnitude of the surface load is $100 \mathrm{MPa}$; solid blue line - damping model, the magnitude of the surface load is $10 \mathrm{MPa}$; cyan dotted line - Maxwell's model, the magnitude of the surface load is $100 \mathrm{MPa}$; red dotted line - Maxwell's model, the magnitude of the surface load is $10 \mathrm{MPa}$ ).

The more pronounced dependence the obtained results have upon the applied loading frequency. Fig. 7 presents temperature rise at the crack tip for the four values of the loading frequency: $10000 \mathrm{~Hz}, 20000 \mathrm{~Hz}, 30000 \mathrm{~Hz}$ and 50000 Hz. It can be seen that the increase in the loading frequency gives approximately twofold increase in the maximum value of the temperature rise at the crack tip. Therefore, the increase in the value of the loading frequency can help to improve the visibility of the crack.

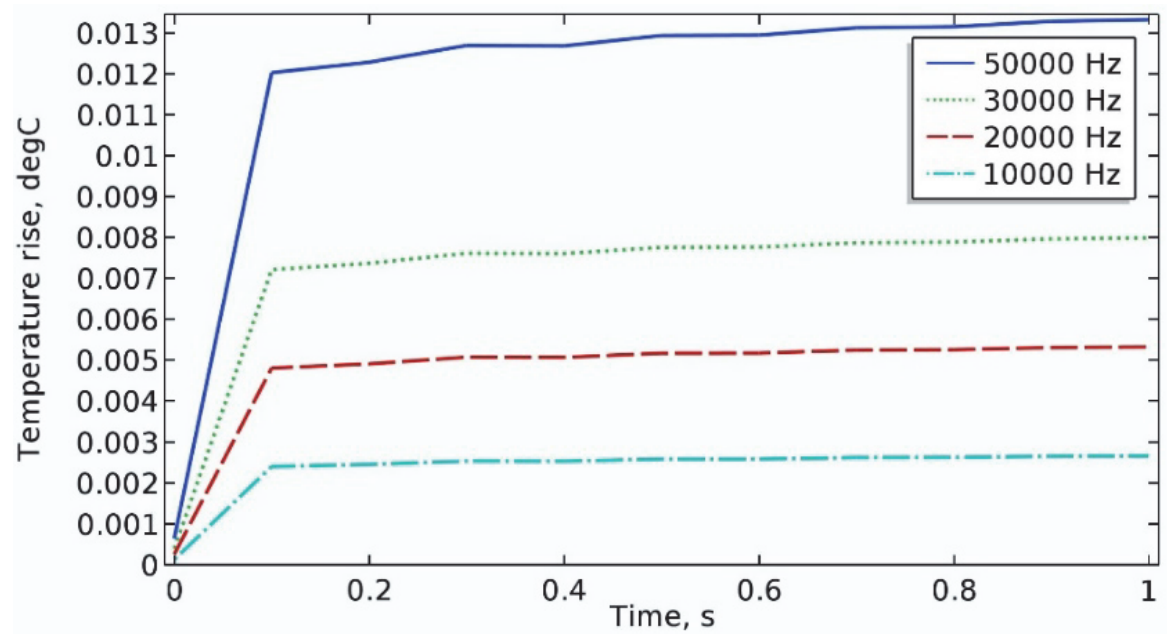

Figure 7: Temperature rise at the crack tip under various frequency loading (solid line $-50000 \mathrm{~Hz}$, dotted line $-30000 \mathrm{~Hz}$, dashed line $-20000 \mathrm{~Hz}$, dash-dot line $-10000 \mathrm{~Hz}$ ).

The way in which the load is applied to the specimen is also affects the visibility of the crack. Figs. 8 and 9 show the distribution of the temperature rise in case when the applied load is directed parallel to the crack plane. In this case, the crack is rotated on 90 degrees with regard to the case presented in Fig. 2. The surface load is applied to the opposite end of the specimen. This kind of loading leads to the stationary wave, whose peak amplitude does not move in space. The crack tip location coincides with nodes of the wave. On the initial stage of the loading process, the crack tip is visible (fig. 8(a), 9(a)). Further loading leads to the heating of the whole specimen (fig. 8(b), 9(b)) and blurring of the temperature localization at the crack tip. The maximum temperature values are in the antinodes of the wave under such loading. The obtained results also shows that Maxwell's model gives more pronounced crack tip compared to the isotropic damping model. 


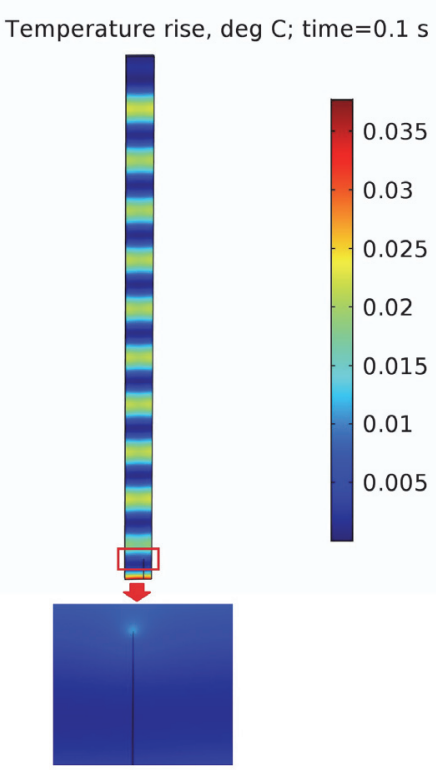

(a)

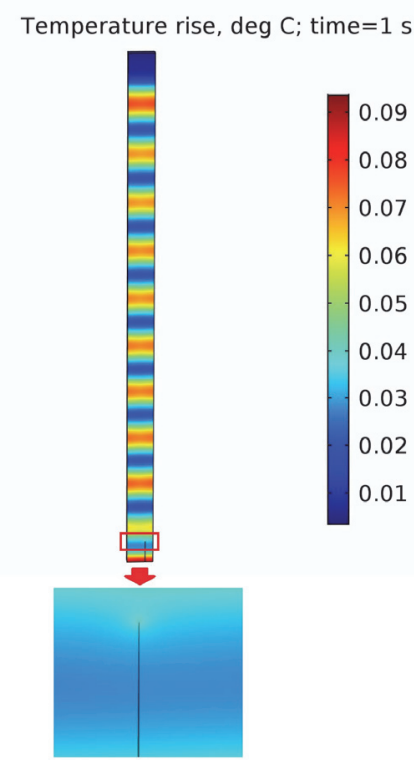

(b)

Figure 8: Distribution of the temperature rise as a result of the ultrasonic excitation by the force parallel to the crack plane calculated by the damping model: (a) $t=0.1 \mathrm{~s}$, (b) $\mathrm{t}=1 \mathrm{~s}$.

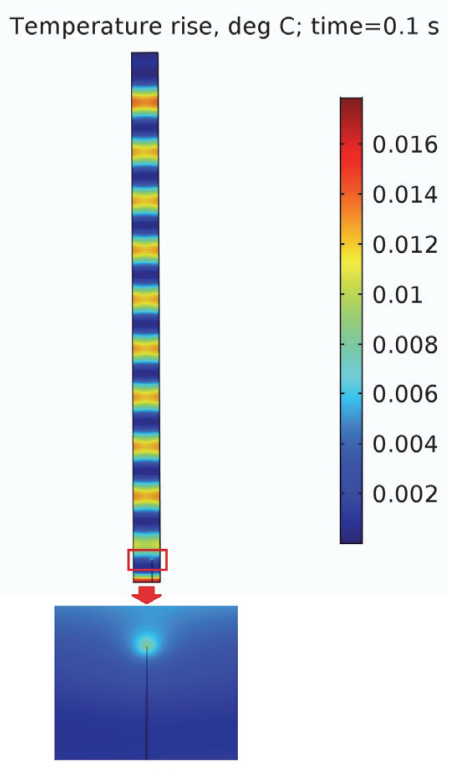

(a)

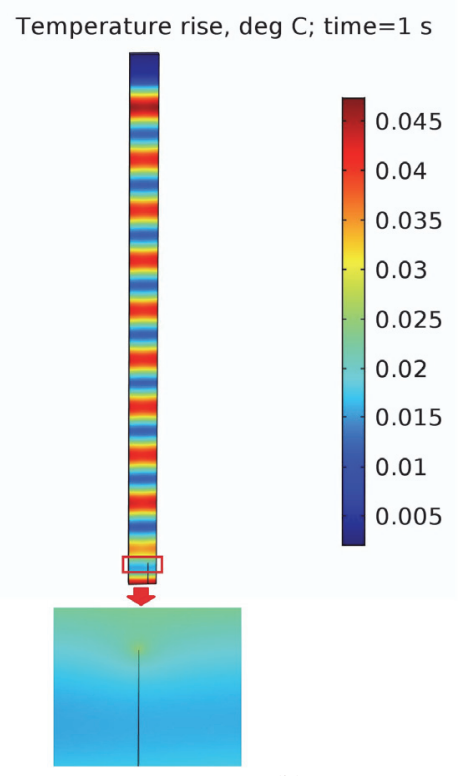

(b)

Figure 9: Distribution of the temperature rise as a result of the ultrasonic excitation by the force parallel to the crack plane calculated by the Maxwell's model: (a) $t=0.1 \mathrm{~s}$, (b) $t=1 \mathrm{~s}$.

\section{CONCLUSION}

$\mathrm{I}$ $\mathrm{n}$ this study, a numerical simulation of a crack detection by an ultrasonic thermography method is presented. The essence of the method is to affect the specimen by a periodic mechanical force with simultaneous recording of the temperature signal. The obtained temperature signal is generated mainly in the area containing cracks and other defects while the surrounding material keeps the same temperature. Local heating in the specimen is related to the dissipative processes arising during the mechanical vibration. In this work, two kinds of models describing energy dissipation and heat generation at the crack tip are considered: material (hysteretic) damping model characterized by an 
isotropic loss factor and visco-elastic Maxwell's model. The application of both models is illustrated by numerical simulation of ultrasonic excitation of the steel specimen with a copper coating containing an edge crack.

The obtained results have shown that the visibility of the crack depends on the loading method. The crack is highly visible when the applied load is perpendicular to the crack plane. Under such loading conditions, the Maxwell's model and isotropic damping model can be considered as equivalent with regard to the temperature rise value. The emergence of the stationary wave blurs the visibility of the crack tip, because the maximum temperature values coincides with the antinodes of the wave. Under such loading case, Maxwell's model gives more visible crack tip compared to the isotropic damping model. Both considered models are sensitive to the values of the material parameters. A sensitivity of the obtained results to the loading magnitude can be observed when the values of the isotropic loss factor and relaxation time value are small enough. The most pronounced is the dependence of the obtained results on the loading frequency. The increase in the loading frequency gives approximately twofold increase in the maximum value of the temperature rise at the crack tip. The optimal conditions for application of ultrasonic vibrothermography include relative high loading frequency and ultrasonic excitation perpendicular to the crack plane.

\section{ACKNOWLEDGMENTS}

his work is supported by the Russian Science Foundation (Grant No. 15-19-10056).

\section{REFERENCES}

[1] Henneke, E.G., Reifsnider, K.L., and Stinchcomb, W.W. (1979). Thermography - an NDI method for damage detection, J. Met., 31, pp. 11-15.

[2] Pye, C.J. and Adams, R.D. (1981). Detection of damage in fiber reinforced plastics using thermal fields generated during resonant vibration, NDT Int., 14(3), pp. 111-118.

[3] Guo, X. and Vavilov, V.P. (2013). Crack detection in aluminum parts by using ultrasound excited infrared thermography, Infrared Phys. Technol., 61, pp. 149-156.

[4] Truell, R., Elbaum, C. and Chick, B.B. (1969). Ultrasonic methods in solid state physics, New York and London, Academic Press.

[5] Sabotkarin Rizi, A., Hedayatrasa, S., Maldague, X. and Vukhanh, T. (2013). FEM modeling of ultrasonic vibrothermography of a damaged plate and qualitative study of heating mechanisms, Infrared Phys. Technol., 61, pp. 101-110.

[6] Plum, R. and Ummenhofer, T. (2011). Structural-thermal finite element simulation of vibrothermography applied to cracked steel plates, Quant. Infrared Thermogr. J., 8, pp. 201-220.

[7] Hiremath, S.R., Mahapatra, D.R. and Srinivasan, S. (2012). Detection of crack in metal plate by thermo sonic wave based detection using FEM, J. Exp. Stroke Transl. Med., 1(1), pp. 12-18.

[8] Solodov, I., Rahammer, M., Derusova, D. and Busse, G. (2015). Highly-efficient and noncontact vibro-thermography via local defect resonance, Quant. Infrared Thermogr. J., 12(1), pp. 98-111.

[9] Findeisen, D. (2000). System dynamics and mechanical vibrations, Berlin, Springer.

[10] Comsol. (2017). COMSOL Multiphysics User's Guide, Version 5.3a.

\section{NOMENCLATURE}
$\rho$ Density;
u Displacement vector;
$t$ Time;
$\sigma$ Cauchy's stress tensor;
f Volumetric force vector;
$F[\cdot]$ Fourier transform of a function; 
$\omega$ Angular frequency;

$i$ Imaginary unit;

$\varphi$ Phase;

C Stiffness tensor;

$\varepsilon^{e l}$ Elastic strain tensor;

$E$ Young's modulus;

$v$ Poisson's ratio;

$\alpha$ Linear thermal expansion coefficient;

$T$ Temperature;

$T_{0}$ Initial temperature value;

$\varepsilon$ Full strain tensor;

$\sigma_{d}$ Deviatoric stress tensor;

$\varepsilon_{d}$ Deviatoric strain tensor;

$G^{\prime}$ Shear storage modulus;

$G$ " Shear loss modulus;

$\tau$, Relaxation time;

$G_{v}$ Shear modulus;

$\operatorname{conj}(\cdot)$ Complex conjugate value;

$Q_{v}$ Viscous mechanical dissipation;

$\eta$ Isotropic loss factor;

$Q_{b}$ Hysteretic damping;

$C_{p}$ Heat capacity under the constant pressure;

$k$ Thermal conductivity;

$Q$ Heat source 